\title{
High data-rate optical communication payload for CubeSats
}

Rödiger, Benjamin, Menninger, Christian, Fuchs, Christian, Grillmayer, Lukas, Arnold, Saskia, et al.

Benjamin Rödiger, Christian Menninger, Christian Fuchs, Lukas Grillmayer, Saskia Arnold, Christoph Rochow, Philipp Wertz, Christopher Schmidt, "High data-rate optical communication payload for CubeSats," Proc. SPIE 11506, Laser Communication and Propagation through the Atmosphere and Oceans IX, 1150604 (22 August 2020); doi: 10.1117/12.2567035 


\author{
High data-rate Optical Communication Payload for CubeSats \\ Benjamin Rödiger*a ${ }^{*}$, Christian Menninger ${ }^{\mathrm{a}}$, Christian Fuchs ${ }^{\mathrm{a}}$, Lukas Grillmayer ${ }^{\mathrm{b}}$, Saskia Arnold ${ }^{\mathrm{b}}$, \\ Christoph Rochow ${ }^{\mathrm{c}}$, Philipp Wertz ${ }^{\mathrm{c}}$, Christopher Schmidt ${ }^{\mathrm{a}}$ \\ ${ }^{a}$ German Aerospace Center (DLR), Institute of Communications and Navigation, Muenchener Str. \\ 20, 82234 Wessling, Germany \\ ${ }^{\mathrm{b}}$ German Aerospace Center (DLR), German Space Operation Center, Muenchener Str. 20, 82234 \\ Wessling, Germany \\ ${ }^{\mathrm{c}}$ Tesat-Spacecom GmbH \& Co. KG, Gerberstr. 49, 71522 Backnang, Germany
}

\begin{abstract}
Space industry has undergone a significant change over the last years. The development moved from large and costly spacecrafts to cost-efficient designs and shorter development times. While the satellites became smaller, the resolution of high compact sensors increased which led to a high data-volume to be transmitted and increasing demands for higher data rates on small satellites. This motivated for a highly compact version of DLR's optical communication payload OSIRIS for small LEO satellites.

DLR's Institute of Communications and Navigation has developed the OSIRIS (Optical Space Infrared Downlink System) program starting with payloads on the satellites Flying Laptop of Univ. of Stuttgart and BiROS of DLR. Combining miniaturization to the flight-proven developments with novel concepts, OSIRIS4CubeSat allows integration in a standard CubeSat bus.

The development of OSIRIS4CubeSat (industrialized under the product name "CubeLCT") is conducted in close collaboration with Tesat Spacecom, DLR's commercialization partner. The first implementation will be demonstrated within the PIXL-1-Mission on a 3Unit CubeSat. Furthermore, OSIRIS4CubeSat (O4C) has been chosen to support scientific missions together with university partners in the field of Quantum Key Distribution (QUBE). In the future, the modular design will enable extensions for optical inter-satellite links.
\end{abstract}

This paper will give an overview about the development of the O4C payload and the current status of the PIXL-1Mission. Furthermore, it will show the adaptation of the payload for the scientific mission QUBE. Besides these projects, the paper will give an outlook for future extensions of the $\mathrm{O} 4 \mathrm{C}$ payload and the necessity of high data-rates in other scenarios such as inter-satellite links.

Keywords: OSIRIS, Free-Space-Optics, CubeSat, Laser-Communication, high data-rate

\title{
1. INTRODUCTION
}

In the previous years, optical satellite communications have gained increasing attention. The applicability of optical links in Space has been shown in a number of application scenarios, such as in inter-satellite-links[1], satellite-downlinks[2-4] and even Deep Space links[5]. More than 10 years ago, the German Aerospace Center DLR started to focus on optical communication from small satellite platforms. Increasing sensor resolution as well as a growing number of smaller satellites raised the interest in this field. While optical communication links on larger satellites are already more or less standard applications and available on the market, miniaturized systems were not yet developed. The program OSIRIS (Optical Space Infrared Downlink System) started with scientific payloads OSIRISv1 on Flying Laptop (University of Stuttgart) with launch in 2017 and OSIRISv2 on BiROS (DLR Berlin) with launch in 2016[6]. OSIRISv1 has a maximum data rate of $200 \mathrm{Mbit} / \mathrm{s}$ and has been used for more than 150 link experiments to DLR's optical ground station in the meantime. OSIRISv2 increased the data rate to $1 \mathrm{Gbit} / \mathrm{s}$ by adding a tracking sensor to the setup that provides precise pointing information to the satellite's orientation control and with that decreases the divergence. Both satellites, Flying Laptop and BiROS are around $120 \mathrm{~kg}$ and are rather large missions compared to CubeSat applications. On basis of the experience from successful space qualification with OSIRISv1 and v2 and the growing number of CubeSat launches, a miniaturized terminal was developed. Especially the challenging limitations on CubeSats for size, weight and power (SWaP) drive the need for a highly compact system design with the largest possible optics for a small divergence and high power efficiency. To increase the flexibility to fit in different CubeSat platforms, the goals were set to a weight

*Benjamin.roediger@ dlr.de; phone +49 8153 28-2944; fax +49 8153 28-2844; dlr.de/kn/en/

Laser Communication and Propagation through the Atmosphere and Oceans IX, edited by Jaime A. Anguita,

Jeremy P. Bos, David T. Wayne, Proc. of SPIE Vol. 11506, 1150604 · (C) 2020 SPIE

CCC code: $0277-786 \mathrm{X} / 20 / \$ 21 \cdot$ doi: $10.1117 / 12.2567035$ 
below $400 \mathrm{~g}$, a size of max. $10 \times 10 \times 3 \mathrm{~cm}$ and a power consumption below $8.5 \mathrm{~W}$ for a data rate of $100 \mathrm{Mbit} / \mathrm{s}$. The development followed two main goals: to create a scientific platform for experiments and demonstrations to enable further projects in the framework of optical satellite communications, as well as a commercial market application via Tesat Spacecom.

\section{OSIRIS4CUBESAT}

The goals of the OSIRIS4CubeSat project are the concept, design, development, test and qualification of a high compact, power efficient laser communication payload, a demonstration mission together with GomSpace as satellite manufacturer and GSOC as satellite operator as well as a preparation for the serial production at Tesat Spacecom's facilities in Backnang, Germany.

\subsection{Project phases of $\mathrm{O4C}$}

The main part of the project is to develop a high compact laser communication terminal for CubeSats. Fitting the whole development from scratch to a quasi-operational demo mission in orbit into one project is very ambitious. To reduce costs and development time DLR decided to follow the so-called New Space approach representing modern development and qualification processes in the CubeSat domain.

The projects' structure was orientated on the NASA Systems Engineering project life cycle but tailored down to the necessary phases according to the New Space approach. The phases of the project were ended with the corresponding reviews as shown in Table 1:

\begin{tabular}{|rl|l|}
\hline \multicolumn{1}{|c|}{ Phase } & \multicolumn{1}{|c|}{ Review } \\
\hline 1. & Concept & Preliminary Design Review (PDR) \\
\hline 2. & Design & Critical Design Review (CDR) \\
\hline 3. & Development & Technical Readiness Review (TRR) \\
\hline 4. & Qualification & Launch Readiness Review (LRR) \\
\hline 5. & Operation & System Functionality Review (SFR) \\
\hline
\end{tabular}

Table 1. Phases and reviews in the OSIRIS4CubeSat project

Phase 1 started with the Computer Aided Design (CAD) of each part and the simulation of the optical system using ZEMAX. The resulting 3D designs allow identifying obstacles in a shortness of time before expensive prototypes or technical demonstrators have to be changed or reproduced. Based on the design, the harsh environmental conditions expected in space and during the launch including the effects on the system could be simulated. Therefore the software ANSYS provides Finite Elements Methods (FEM) to analyze mechanical stress and deformations caused by vibrations during the launch and temperature gradients in space. The advantage of using FEM in phase 1 and 2 is to minimize the risk of failing qualification processes described in chapter 6 . If an issue occurs during the simulations the design can easily be adapted. This starts an iterative process which causes much less effort as if the issues would have been realized during phase 3 or 4 . A detailed description of the phases follows in the next chapters.

\section{CONCEPT}

The outcome of the OSIRIS4CubeSat project is a high compact, power efficient laser terminal, motivated by the limitations in nano- and picosatellites for Size Weight and Power (SWAP). Its size of only 0.3 Unit (U) of a CubeSat, together with following the mechanical standard of PC/104[7] allows the integration in most of the common CubeSat busses. O4C can transmit data up to $100 \mathrm{Mbit} / \mathrm{s}$ from Low Earth Orbit (LEO) to the ground, by consuming less than $8.5 \mathrm{~W}$ electrical power. Preliminary link throughput estimations with a typical OGS network show, that with a data rate of $100 \mathrm{Mbit} / \mathrm{s}$, an average volume of $4 \mathrm{GByte} /$ day can be transmitted to ground, which equals hundreds of high resolution images.

The fundamental idea of $\mathrm{O} 4 \mathrm{C}$ is to use the Printed Circuit Board (PCB) of the electronical system as the optical bench. Integrating the optical parts directly on the PCB allows maximum compactness. To avoid misalignments due to thermal deformation a copper-invar-copper (CIC) PCB was considered. 
To realize optical communication on a CubeSat, the orientation of the CubeSat towards a ground station is essential and with this the requirements for the satellite's attitude control system. To keep the requirements for the absolute pointing area in a range of $\pm 1^{\circ}$, the OSIRIS payload is equipped with a fast steering mirror to compensate residual tracking errors. Most CubeSats are able to point with an accuracy of $< \pm 1^{\circ}$. Thus, $\mathrm{O} 4 \mathrm{C}$ is designed to compensate this inaccuracy to be able to fly on common nano- and picosatellite platforms. The system is capable of correcting the Point-Ahead Angle (PAA) due to the signal propagation time.

To establish a link, O4C uses a Pointing, Acquisition and Tracking (PAT) system. For this, the Optical Ground Station (OGS) sends a laser beam (beacon) with a relatively large divergence to the satellite, the sensor inside of O4C acquires the beacon, measures the angular offset and sends this offset to an actuator, which compensates the error. A 4-quadrant diode (4QD) is used as the sensor and a Fine Steering Mirror (FSM) is used as the actuator. The transmission beam is coupled into the exact same optical path as the beacon to guarantee that it hits the OGS. This Fine Pointing Assembly (FPA) inside the terminal is able to cover the field of regard of $\pm 1^{\circ}$ ex aperture. A control loop with a bandwidth of $200 \mathrm{~Hz}$ allows the FPA to be that accurate that divergence can be lowered down to the refraction limit, which leads to the highest possible power density on ground.

\section{DESIGN}

Right from the start of the development the potential of future applications of O4C like Quantum Key Distribution (QKD), inter-satellites links (ISL) or just higher data rates were considered. Therefore DLR decided to develop the payload in a way that it is built as system of several, definite subsystems, to avoid complete new developments for future projects. With the modular design it is possible to exchange or adept single subsystems without reconstructing the whole systems structure. For example the transmission subsystem can be easily exchanged by another laser source without changing the entire system.

Laser terminals usually have a mechanically separated optical and electronical subsystem. To achieve a high compact design the optomechanics are integrated directly on top of the electronics mainboard. FEM analysis in the design phase showed that the parts move for high and low temperatures so much that the alignment could not be guaranteed, even though CIC was considered as PCB material which has a Coefficient of Thermal Expansion (CTE) close to zero. Hence DLR decided to change the concept slightly and pack the optical parts in two aluminum blocks, the optomechanics. Because of a CTE mismatch CIC is not necessary anymore and common PCB material can be used. Figure 1 shows a $\mathrm{CAD}$ model of the final payload.

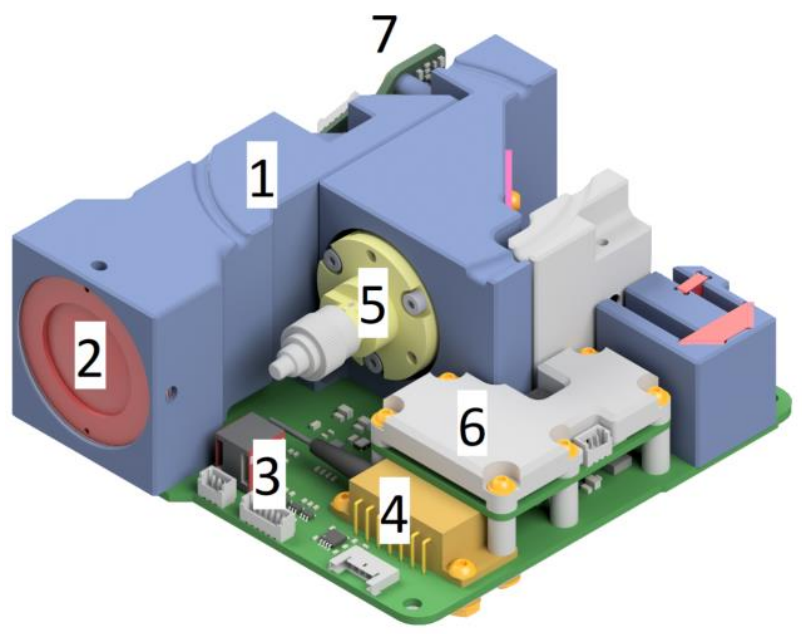

Figure 1. 3D model of OSIRIS4CubeSat Payload

The blue parts are the optomechanics (1) which are the mechanical mounts and housings for the optical elements highlighted in light red (2). Below in green you can see the electronics mainboard (3). The transmission system consists of the laser source in orange (4) and the transmit collimator in yellow (5). The driver electronics sits on the small, green Printed Circuit Board (PCB) with a passive cooling element on top in gray (6). The receiver sensor is located on a separated PCB at the back side of the terminal (7). 
The main part of the system is the optical path. As learned from past projects the alignment process of transmission and receiving beam, so called Tx-/Rx-alignment gets exponentially more complicated as the degrees of freedom increase. Therefore, DLR decided to build a compact optomechanics which integrates all optical components into two aluminum blocks, without any adjustable mechanisms. Thus, DLR had to completely rely on the accuracy of the manufacturer of the optomechanics. A tolerance analysis of the optical system which considered the manufacturing tolerances showed that no further alignment mechanisms are necessary. For the Tx-/Rx-alignment a collimator with a tumbling plate was evaluated, which provides the only necessary degree of freedom for the alignment.

In the CubeSat sector several competing standards can be found. One of the most common standards is the PC/104 standard. The mechanical dimensions and the mounting positions of the $\mathrm{O} 4 \mathrm{C}$ payload are compatible to this standard. The PC/104 standard also defines a 104 pin-connector where the name of the standard comes from. As this connector is relatively large compared to other solutions DLR decided to use compact Commercial Off The Shelf (COTS) connectors which are compatible to most harnesses used in CubeSats. Adapter boards from PC/104 to the interfaces used in O4C are also commercially available.

$\mathrm{O} 4 \mathrm{C}$ is equipped with an own microcontroller. This microcontroller is mainly responsible for the control loop of the FPA inside the system. Every resource intensive calculation like coding or data handling is outsourced to the satellite bus. Telemetry and Telecommands (TM/TC) are sent to/from O4C via an UART interface. The downlink data itself is transmitted via a Low Voltage Differential Signal (LVDS). To be able to reprogram the microcontroller in space, a Joint Test Action Group (JTAG) interface is implemented.

\section{DEVELOPMENT}

To minimize the costs and development time, two identical models of $\mathrm{O} 4 \mathrm{C}$ were built in phase 3, one Engineering Qualification Model (EQM) and one Flight Model (FM). Having two identical models allowed to perform the whole test and qualification process with the EQM and building up the FM in parallel. The preparations in phase 1 and 2, allowed starting the assembly of both models straight after the CDR. Using COTS components enabled a development in a short time as long lead items could be avoided or identified in earlier phases. Even though the components are not space qualified they were selected with the view on the harsh environment. The evaluation was focused on components which already have a heritage in a harsh environments like in automotive. They are not qualified for space but it is proven that they survive in hard environmental conditions.

Using COTS components has further advantages that they are easily replaceable. Considering electronic parts with the same footprints allows replacing them when a non-conformance occurs or results of a functional test show that the electronics have to be changed. Without changing the whole circuit design the parts can easily be replaced.

The modular design of $\mathrm{O} 4 \mathrm{C}$ helps to test single subsystems independent from the development state of the other subsystems. Characterizing the optical system and aligning the beams already can be done while in parallel the software is developed or the electronics are built up. Each system can be tested and verified independently and afterwards integrated into one payload.

\subsection{O4C on CubeL}

$\mathrm{O} 4 \mathrm{C}$ will be demonstrated for the first time in orbit on a $3 \mathrm{U}$ CubeSat from GomSpace, named "CubeL". A system overview of CubeL can be found in Figure 2. 


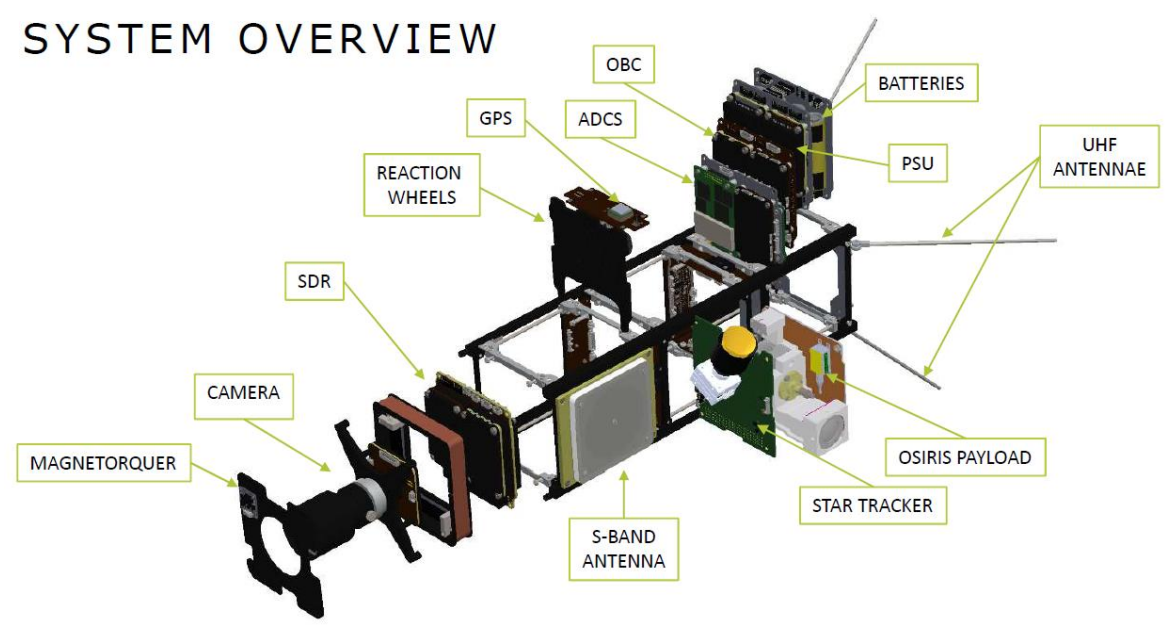

Figure 2. System overview of CubeL

The main goal of the PIXL-1 mission is to demonstrate the technology of O4C. Nevertheless the ability of O4C to provide a whole quasi-operational transmission shall be demonstrated as well. Therefore CubeL is equipped with a camera to take pictures of the earth which will be transmitted via $\mathrm{O} 4 \mathrm{C}$ to the ground.

With its high accuracy Attitude Determination Control System (ADCS) consisting of a high-performance star tracker, Global Positioning System (GPS), reaction wheels and magnetorquers, CubeL is able to realize a pointing stability of better than $\pm 1^{\circ}$. This is sufficient for the FPA of O4C to compensate.

For the main communication with the satellite, conventional Radio Frequency (RF) systems are used. By adding a Software Defined Radio, CubeL is able to transmit and receive data over Ultra High Frequency (UHF) and S-band transceiver. The commanding of the satellite will be done by the German Space Operation Center (GSOC) in Oberpfaffenhofen.

As mentioned in chapter 4, most of the calculation processes are outsourced to the satellite bus. In this mission CubeL is equipped with a Field Programmable Gate Array (FPGA) from GomSpace "Z7000". Parts of the software, especially for the coding and the data handling were developed by DLR. Therefore it had to be implemented on the Z7000. To decrease the implementation effort and reduce the integration time of $\mathrm{O} 4 \mathrm{C}$ in CubeL, a "FlatSat" was procured. The FlatSat includes identical replicas of the subsystems of CubeL which have interfaces to $\mathrm{O} 4 \mathrm{C}$ or are necessary for these. With having a FlatSat during the development all interfaces and software implementations could be tested in advance.

The advantage of using a FlatSat could be seen when the $\mathrm{FM}$ of $\mathrm{O} 4 \mathrm{C}$ was delivered. The payload was integrated straight forward into CubeL without any interface issues. GomSpace could directly start with the integration, testing and qualification of the satellite. 


\subsection{Current status of PIXL-1}

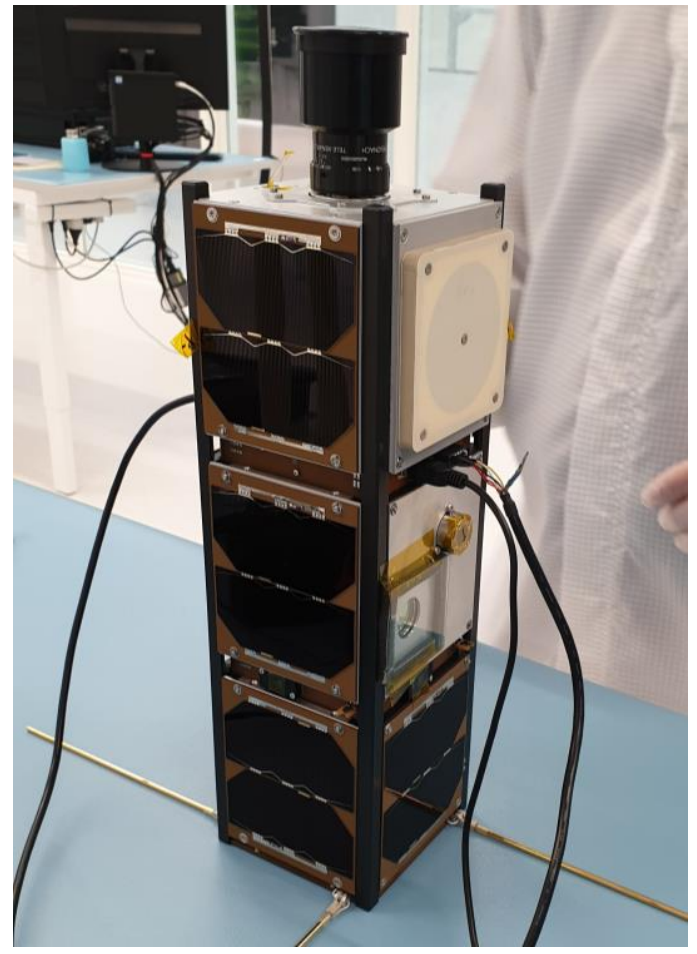

Figure 3. CubeL

Figure 3 shows the fully integrated satellite CubeL. In the second unit on the right side, the aperture of O4C can be seen, covered by an optical filter plate. The reduced and full functional tests were successfully done, so that GomSpace can perform the last environmental tests on CubeL. The qualification will be described in chapter 6.2 in more detail. The launch is planned for the second half of 2020.

\section{QUALIFICATION}

New Space is a move in space industry of the last years to enable faster and cost efficient access to space. Mainly driven by startup companies the philosophy of New Space is to tailor down long-running development and qualification processes known from traditional space missions. Traditional space uses special space qualified components which are have to undergo strict qualification processes. The New Space approach allows to use COTS components and adept the qualification processes accordingly.

DLR-IKN also follows the New Space spirit in their OSIRIS projects. As described in chapter 4, DLR uses COTS components in their developments to avoid costly space qualified components. The idea is to test and qualify as much as necessary but not more than reasonable. The qualification processes in the O4C project are still based on the standards of the European Cooperation for Space Standardization (ECSS) but are tailored down to the minimum and reasonable needs. Hence, DLR does not perform every test suggested by the ECSS. Tests are only done to ensure that the payloads functionality in the harsh space environment is guaranteed and that the COTS components survive in space. To reduce complexity, cost and time of the qualification all tests are done on system level and not on component level. ECSS distinguishes between qualification and acceptance tests. Qualification tests are done with an EQM while acceptance tests are performed with a FM. The tests are basically the same but the qualification loads are higher than the acceptance loads. This procedure shall prevent the FM from stressing it with high loads even before the launch. On the other hand passing of the qualification can be assured by using an identical model, the EQM. This procedure is also followed in the O4C project. 


\subsection{Qualification of $\mathrm{O} 4 \mathrm{C}$}

ECSS describes and recommends a huge amount of tests and qualification steps for space missions. Following the New Space approach DLR decided to do only the absolute necessary tests with the scope on the PIXL-1 mission. To reduce the complexity all qualification tests are done with the EQM on system level and not on component level. After a detailed risk assessment the qualification for $\mathrm{O} 4 \mathrm{C}$ could be tailored down to three mandatory tests:

1. Vibration

2. Thermal Vacuum

3. Radiation

During the launch, the rocket produces strong vibrations which are transferred to all including satellites and systems. To test if a payload survives these forces ECSS recommends to do vibration tests in advance. The qualification tests shall represent the maximum loads that the launcher produces including a margin of safety. Therefore four different types of tests have to be performed:

- Modal frequency

- Sine vibrations

- Random vibrations

- $\quad$ Shock tests

The modal vibration test measures the first harmonic frequency of the payload. It has to be performed before and after every sine and random vibration test. Sine and random vibration tests represent the expected vibration spectrums of the launching rocket. The qualification is passed if the first harmonic is above the first harmonic of the considered launcher, the modal frequency does not change by more than $10 \%$ after another test and the payload is not damaged. All these tests have to be done in every axis which leads to 15 runs in total. Additionally ECSS describes shock tests as they can be expected in a launch as well, because of stage separations for example.

The recommended loads are standardized in the General Environmental Verification Standard (GEVS) by NASA. The loads mentioned in the GEVS are so high that a payload qualified by this standard is able to fly on every launcher available on the market. O4C has very sensitive optical and mechanical elements inside. Qualifying for GEVS loads would mean to face the EQM with much higher loads than every considered launcher for the PIXL-1 mission can produce. There would be a high risk that the EQM could get damaged by overqualifying the payload. Thus DLR decided to reduce the loads according to the highest loads of all possible launchers including a margin of safety. Furthermore none of the possible launcher creates shocks during launch which is why shock tests were not performed with O4C. For future missions a delta qualification with GEVS loads is considered after finishing the project.

When the satellite is in orbit it has to face harsh environmental conditions like ultra-high vacuum and extreme temperatures. These conditions can be recreated on earth in a Thermal Vacuum Chamber (TVAC). The values of the temperature and the pressure are not given by the standard. They have to be evaluated according to the expected orbit. ECSS states one cycle for non-operational temperature and seven cycles for operational temperature tests. Figure 4 shows the cycles for $\mathrm{O} 4 \mathrm{C}$. 


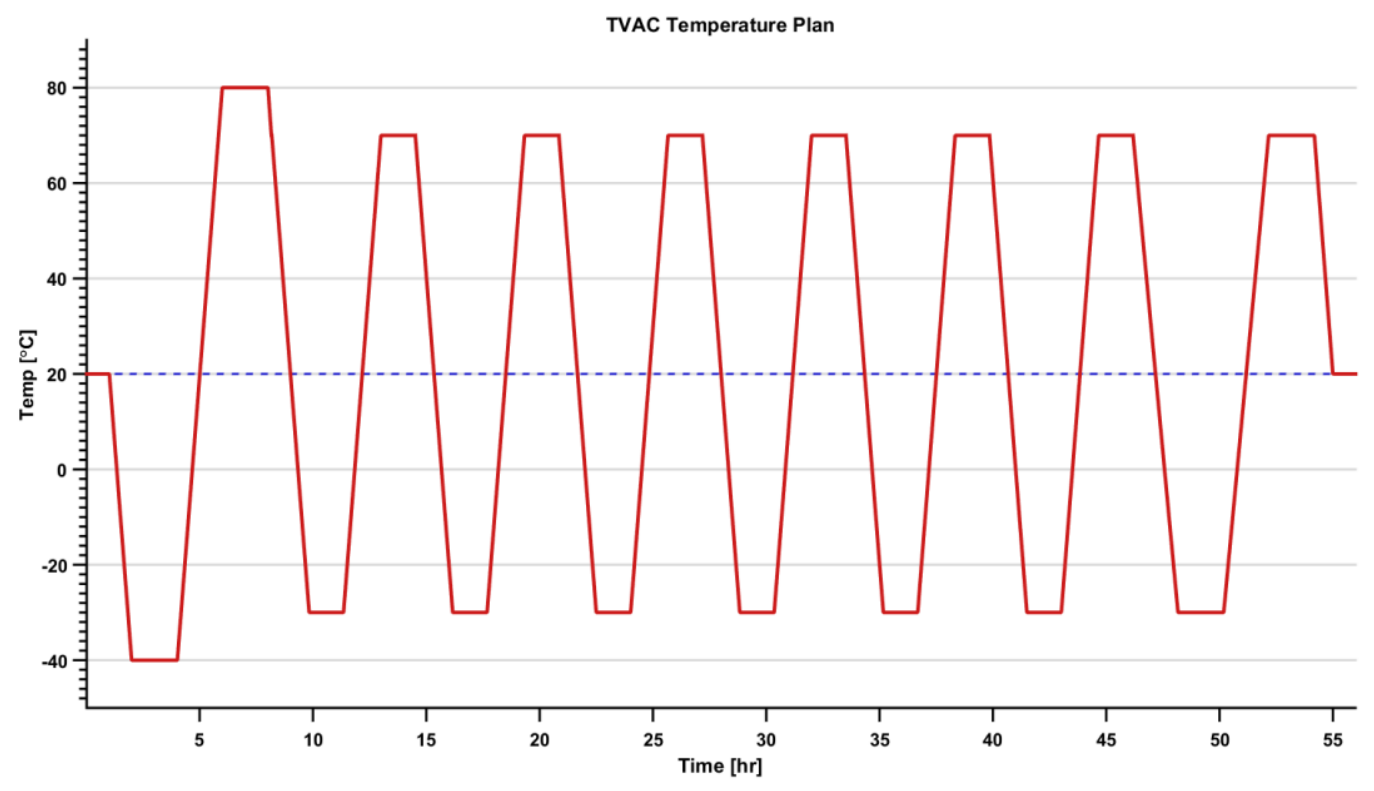

Figure 4. TVAC test cycles for O4C

According to ECSS the TVAC test is passed with a positive full-functional test at every extreme temperature. O4C did not only stick to this standard it went beyond. Additionally OCS defined own pass and fail criteria. Beside the functional tests, the deformation of the EQM due to the extreme temperatures has to be that low that the angle between transmission (tx) and receiving (rx) beam does not exceed a defined threshold. Furthermore this tx-/rx-alignment was measured during one upcycle to verify that the alignment does not exceed the threshold between the extreme temperatures.

Vibration and TVAC test were simulated by the in chapter 2.1 mentioned FEM. The qualification tests verified the results of these analyses. Simulating radiation effects would theoretically be possible but the effort is significantly higher than the qualification test itself. CubeL will be operated in LEO where the electron, neutron and proton radiation is relatively low. Nevertheless a Total Ionization Dose (TID) has to be considered. The total dose depends on the orbit and the expected lifetime of the satellite. ECSS states to test every single part separately and measure its functionality before and after the radiation. To reduce the test complexity a TID test was performed on payload level with the EQM. The device was placed into a radiation chamber with a cobalt-60 source to be radiated with the dose which is expected during the PIXL-1 mission. The pass and fail criteria was a full functional test before and after the radiation.

After the radiation test of $\mathrm{O} 4 \mathrm{C}$ an issue occurred that one electronic part did not work anymore. A second test with a similar electronics board confirmed that this specific part fails after radiation. In a third test a test PCB was radiated which contained different types of the failed part from different manufacturers with several parts of each. This allowed the selection of a replacement component compatible with the radiation requirements. The use of COTS components with common footprints allowed a straight forward change of the part to a one from a different manufacturer without any changes in the board or the circuit design. Even though three tests had to be performed it was a lot faster than analyzing every single part with radiation simulation software or testing every part for radiation before building the EQM.

\subsection{Qualification of CubeL}

The EQM of O4C was tested with qualification levels. To not stress the flight hardware before the launch the tests with CubeL including the FM of $\mathrm{O} 4 \mathrm{C}$ are performed on acceptance levels. Building an EQM of the entire satellite was not considered as it would have increased the costs of the project. The systems GomSpace uses in their satellite have a high heritage and are all fully qualified. Additional tests on qualification levels are not necessary. Nevertheless the same tests as described above have to be performed with the satellite but with reduced loads on acceptance levels. Excluded from these tests is the radiation test. As the radiation test is a TID test, radiating parts before sending them into space, where they would gather the same dose on top over their lifetime has to be avoided. 
One additional test which is recommended by ECSS is the Electromagnetic Compatibility (EMC) test. Because of its high complexity it was agreed that the EMC test is done with the whole satellite including O4C.

\section{OPERATION}

CubeL will be operated by the German Space Operations Center (GSOC), which is embedded within the DLR Institute for Astronaut Training and Space Operations and has operated a large variety of satellites for over half a century, with recent activities ranging from unmanned low- and medium earth orbiting earth observation and science missions (e.g. TanDEM-X, GRACE Follow-on, Eu:CROPIS), over geostationary communication and navigation satellites (e.g. EDRSC, HAG-1) to human spaceflight (e.g. ISS-Columbus). All satellites operated at GSOC make use of institutionally standardized communication protocols such as the CCSDS frame (132.0-B-2) and packet standards (133.0-B-1) and the ECSS packet utilization standard (E-ST-70-41C). Over the past two decades this commonality enabled GSOC to develop ground segment system architectures and tools which can be shared and reused by many projects with only minor adaptions. As a result these concepts and tools have been tested excessively and exhibit a high level of maturity, which form the basis of GSOC's multi mission (MUM) environment. This approach has proven to be cost-effective not only concerning software development and system maintenance but also for operations. Subsystem engineers and command operators have deep knowledge about features and functionality of MUM systems, so they are already familiar with the available toolset. This supports them in their effort to efficiently prepare and execute a mission.

Building upon this experience, GSOC's involvement in the DLR internal CubeL mission began in late 2017 with a request for proposal for an operational concept. Boundary conditions were to keep cost low, appropriate for the small size of the mission and to be ready for launch in September 2018, as originally scheduled. LEOP would be performed by GomSpace and GSOC would assume operational control of the spacecraft after a successful handover.

CubeL will be the first COTS cubesat to be operated by GSOC, which in itself poses a few challenges: Cubesats commonly operate in the UHF-band spectrum and the satellite's native communication protocol is the Cubesat Space Protocol (CSP), which is an open source protocol developed and maintained by the University of Aalborg, Denmark and has been adapted and extended by GomSpace. Both technologies had yet to be supported by GSOC[8].

The accepted proposal foresees two mission phases E2a and E2b, which will be briefly presented below. CubeL spacecraft operations will conclude with EOL activities in phase F.

\subsection{Phase E2a: UHF-Band Ground Segment}

The primary mission objective of CubeL is the successful in-orbit demonstration (IOD) of the O4C module's downlink capabilities and reliability. In order to establish operational readiness within the limited designated timeframe, whilst keeping risk as low as possible a CSP compatible COTS UHF ground segment was procured and integrated into the GSOC facilities. The long operational heritage of GomSpace ground segment hardware and software promise minimal setup times and all components have been proven to work reliabily out of the box.

During phase E2a, CubeL will be operated only via UHF utilizing the GomSpace NanoCom AS100 dual Yagi antenna, GS100 dual-radio ground station transceiver, and the MS100 mission computer hosting the CSP monitoring and control system. These components form the baseline for the E2a ground segment, which is further augmented by GSOC MUM components as described below. GSOC security policy requires external hardware and software to be isolated in separate networks and thus may not be integrated into the MUM environment directly.

Within this setup, CSP is the prevalent protocol used for communication between ground segment components and the space segment. The "CSP-Terminal" software is also referred to as GomSpace Shell (GOSH), which is the main TMTC interface used for operating the spacecraft. Further optional software additions include a telemetry visualization solution called "GSWeb", which is operated in parallel to the GSOC wide established "Satmon". Incoming telemetry beacons will be preprocessed by the CubeL Offline Processing System (COPS) for ingestion in Satmon. Beacons contain configurable lists of parameter values, which can be decoded by COPS and Satmon using a carefully crafted SCOS-2000 database. With proper enhancements this MIB can be reused in later mission phases. Preceding the usage in phase E2b, Satmon can draw on existing network infrastructure for remote data delivery to Satmon@ Home as well as previous user experience of all GSOC personnel and most principal investigators, requiring only little training. Further, the concurrent usage of GSWeb and Satmon increases redundancy in phase E2a and allows for early validation efforts important for phase E2b. The E2a ground segment setup will serve as a backup during phase E2b. 
For the provision of commanding input, an established MUM recommendation workflow of OpsWeb is applied, which incorporates an analogue review and signature process. Input for routine payload operations is processed automatically and combined with flight dynamics data via the LCT-Merger, resulting in procedures ready to command. Experience has shown that procedural commanding reduces the risk of commanding errors significantly.

Figure 5 highlights custom software components required for CubeL phase E2a UHF ground station systems to interface with GSOC MUM.

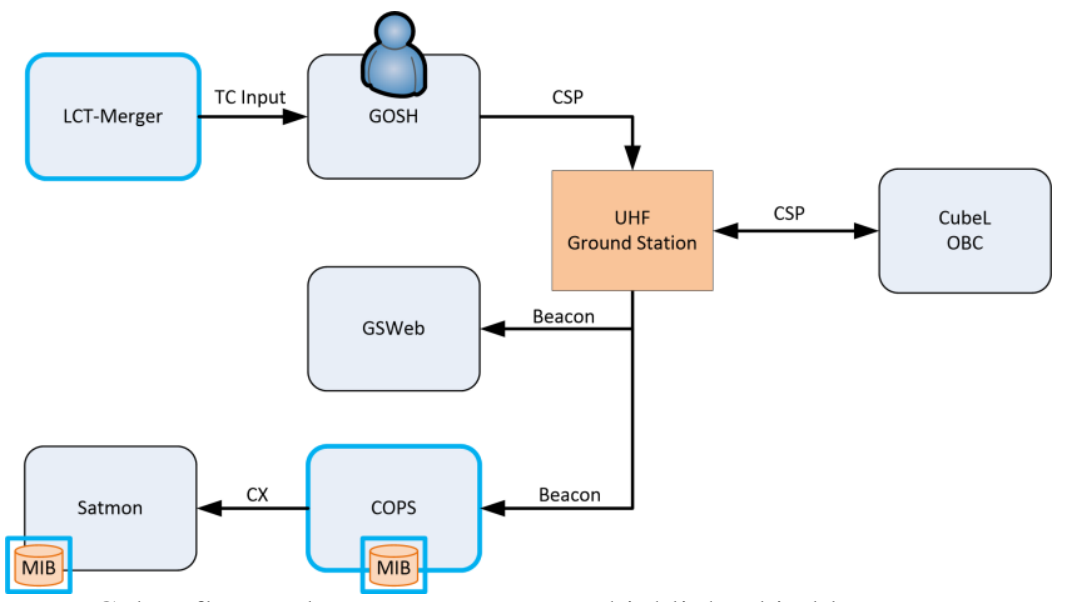

Figure 5. Phase E2a TMTC data flow and custom components highlighted in blue

During phase E2a the primary O4C IOD mission objective is performed. In parallel, the ground segment design for phase E2b will be implemented, not interfering with ongoing operations. Upon completion operations will switch to the S-band based ground segment, while the interface to principal investigators remains the same, thus making the transition transparent to the end customer.

\subsection{Phase E2b: S-Band Ground Segment}

The phase E2b ground segment design aims to extensively utilize existing GSOC MUM capabilities with as little custom additions as possible to accommodate the CubeL missions. All S-band communication between space and ground shall be performed using CCSDS protocols.

For operators, the realtime TMTC interface with all GSOC MUM missions is GECCOS [9], an MCS system based on ESA SCOS-2000, which is developed and maintained in-house. For telemetry visualization and analysis Satmon has been established as the tool of choice for operators, subsystem engineers, and principal investigators (PI) alike as it provides intuitive access to both realtime data with very little delay and large amounts of offline data with low retrieval latencies. The Multimission Offline Processing System (MOPS) handles the processing of recorded satellite telemetry which is excluded from the realtime data transmission channel and forwarded after a contact due to bandwidth limitations of some remote ground stations. Over the past decades the DLR ground station network has grown to a global network of ground station service providers and includes DLR operated sites in Weilheim, Germany (WHM) and O'Higgins, Antarctica (OHG), which allow for GSOC's fault tolerant and highly flexible ground segments, whereas baseline for CubeL S-band operations is WHM ground station.

Figure 6 highlights the custom software components required for CubeL to interface with GSOC MUM. For initial ingestion of novel commands "GOSH2SSF" handles the conversion from GOSH CSP commands to GECCOS Saved Stack Files (SSF). For GECCOS to be able to encode and decode telemetry and commands it needs a description of all packets and parameters in form of the Mission Information Base (MIB), which is an extended variant of the telemetry database developed by GSOC during phase E2a.

By design it is intended for the CCSDS space packet protocol and is able to support the ECSS packet utilization standard (PUS). The CubeL system engineers aim to use the versatility of the MIB for it to serve as an adapter for the cubesat space protocol, allowing GECCOS to talk and interpret CSP in realtime. Within the GSOC MUM environment many tools require a MIB to decode, process, and visualize both realtime and offline telemetry. 
Despite the conversion from UHF- to S-band based operations, the interface to PIs does not change. The ground segment design intends to make the transition as smooth and transparent as possible to not cause service outages, data gaps or any other inconvenience within the customer experience. Overall the presented ground segment fulfills the technical and budgetary design goals.

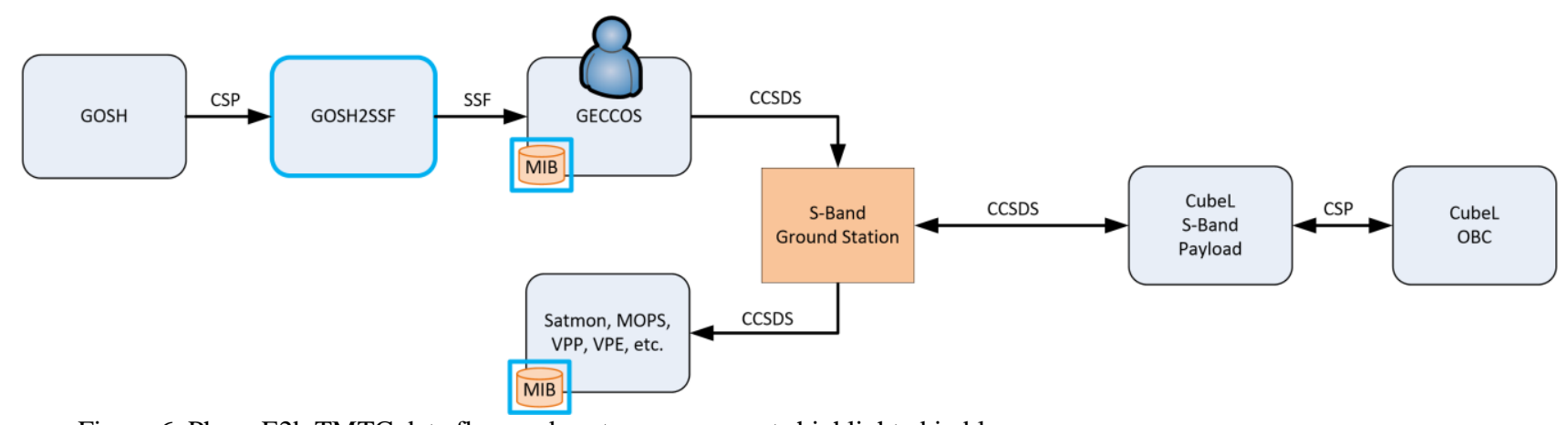

Figure 6. Phase E2b TMTC data flow and custom components highlighted in blue

\section{ADAPTIONS OF O4C AND FUTURE MISSIONS}

One major goal of the OSIRIS4CubeSat development was a modular design with standard interfaces that allows adapting the payload to mission needs. For future applications, OSIRIS will be extended by additional features and performance that will be presented in this chapter. QUBE is a development towards test of Quantum Key Distribution (QKD) techniques together with scientific partners. With OSIRIS-1G the data rate will be increased to $1 \mathrm{Gbit} / \mathrm{s}$ and a higher transmit power to fulfil mission needs of upcoming projects. In OSIRIS-L the payload is further extended with an optical receive channel to work in constellations as optical inter-satellite link (ISL). In scenarios, where optical connections are not always possible, also concepts with hybrid systems, consisting of RF- and optical links, are investigated.

\subsection{QUBE}

Beside the demand for ever higher data rates, there is the need for secure communications. Where state of the art encryption technologies are well established and provide a sufficiently high security for sensitive data for most of the applications, particular fields need an even higher security. Therefore the research association "QUBE" aims at the development of key technologies for secure data communications based on quantum key distribution. Together with other research and industry partners, DLR contributes with an optical terminal for free space coupling of the quantum communications payloads and also for classical communications downlink.

The adaption from O4C to QUBE was mainly driven by the change of the optical system due to the use of multiple wavelengths in the transmitter system. The mechanical and electrical construction was adopted without major changes so that the effort for qualification procedure is kept minimal. A modal analysis is performed to simulate if critical loads appear due to the slightly changed mass distribution. The lens system of $\mathrm{O} 4 \mathrm{C}$ had to be replaced by an achromatic one which changes the number of elements, the material and thus also the mass. A combination of an infrared chalcogenide glass with a heady flint glass showed to have the best performance. In particular the angular displacement of the beams for each wavelength exiting the optical system needs to be as small as possible to hit the ground station. As parts of the system are also used for the tracking system, it needs to be optimized for spot shape at the tracking sensor.

The laser signals of the other payloads are combined with the laser communications signal of QUBE within a fiber network based on thin film filters. A compromise between bend loss and cut off frequency was done for the choice of the output fiber which transports the laser signals to the optical terminal.

\subsection{OSIRIS-1G}

OSIRIS4CubeSat will demonstrate a data rate of $100 \mathrm{Mbit} / \mathrm{s}$. For applications in upcoming missions, this might not always be sufficient and raise the need for higher data rates. The reuse the qualified payload as much as possible, the OSIRIS4CubeSat payload will be extended by additional components and without changing the base development.

To increase the data rate without increasing the size of the optomechanics, the optical transmission power will be increased. While OSIRIS4CubeSat used a directly modulated laser diode, the change to $1 \mathrm{Gbit} / \mathrm{s}$ requires a setup with a 
modulated seed laser and an optical amplifier. This setup has been demonstrated on Flying Laptop and BiROS already with a miniaturized component that can be reused here and provides $1 \mathrm{~W}$ of optical output power.

The channel coding and data handling with $1 \mathrm{Gbit} / \mathrm{s}$ gets more challenging compared to the lower data rates. While the satellite's $\mathrm{OBC}$ has been used for channel coding up to now, the extension to $1 \mathrm{Gbit} / \mathrm{s}$ requires a own processing unit with a mass memory to buffer the mission data, apply the channel coding and read it from the memory for downlink transmission.

A demonstration mission is envisaged for 2021.

\subsection{OSIRIS-L}

Besides higher data rates, optical Inter-Satellite Link (ISL) are a major driver of the CubeSat development at the moment. Different constellations with high numbers of satellites are already launched or are about to be launched soon. A key feature of those constellations is a possibility to exchange data within the constellation with high data rates over long distances. Therefore, optical communication can help to overcome current limitations in mission planning.

While OSIRIS4CubeSat already uses an experimental optical uplink channel, this will not be sufficient for bi-directional and high data rate communication within a constellation. Therefore, the optical system will be extended by an additional receive path, leading to a dedicated data receiver in addition to the tracking sensor.

With an unchanged diameter of the optical system, OSIRIS will be able to transmit data fully bi-directional between two satellites with a data rate of $100 \mathrm{Mbit} / \mathrm{s}$ over a distance of $1500 \mathrm{~km}$.

A demonstration mission is envisaged for 2022.

\subsection{Hybrid Links}

Not all applications are perfect for only one transmission technique. In scenarios with limited availability of optical link, a hybrid link together with an RF-system can be beneficial for the mission. In such a setup, two different link technologies are combined with different strength: while optical communication provides high data rates, it can suffer from limited availability in some areas or scenarios. This limitation can be overcome with RF-communication with a high availability but rather lot data rate. The combination allows using a system with high availability and high data rate whenever possible.

To have an efficient system, the transmitter side is equipped with a processing unit which estimated the channel quality of optical and RF-link by representative parameters and prioritizes the optical link with the high data rate. In case of a drop in channel quality for the optical link, the processing unit directs the data towards the RF-link which can bridge the gap in the optical link.

\section{SUMMARY AND OUTLOOK}

This paper described the system and mission concept of the OSIRIS4Cubesat payload developed by DLR's Institute of Communication and Navigation. The terminal is marketed under the name CubeL by Tesat Spacecom. It is a highlycompact optical communication terminal and occupies only 0.3 Cubesat-Units $(0.3 \mathrm{U})$ and has a power consumption of $8.5 \mathrm{~W}$. It is capable of transmitting data with $100 \mathrm{Mbit} / \mathrm{s}$.

The demonstration mission PIXL-1 and the satellite bus have been described as well. A 3-Unit cubesat is used. This enables the accommodation of a camera payload, such that the system can be used for an end-to-end demonstration ranging from image acquisition, over optical transmission from Space to Earth, to processing the images on ground. The PIXL-1 mission is planned for launch in the $2^{\text {nd }}$ half of 2020.

The OSIRIS4Cubesat development is based on the so-called New Space approach. Therefore, traditional development qualification processes have been tailored to the essential parts. Furthermore, COTS components are used. This approach allows the use of COTS components with a high performance and minimizes development time, resulting in a costeffective process. However, the qualification must be done with appropriate care, and one must be prepared to replace components in case some do not pass the qualification tests.

Throughout the next years, it is planned to use OSIRIS4Cubesat as platform in order to demonstrate further optical communication technologies. Scope of these experiments may be, for instance, quantum key distribution, inter-satellitelinks, or the demonstration of higher data rates from satellite to ground. 


\section{ACKNOWLEDGEMENT}

The authors would like to thank Fabian Rein, Anil Morab Vishwanath, René Rüddenklau and Philipp Biller for their support during the development.

\section{REFERENCES}

[1] D. Tröndle, P. Martin Pimentel, C. Rochow, H. Zech, G. Muehlnikel, F. Heine, R. Meyer, S. Philipp-May, M. Lutzer, E. Benzi, P. Sivac, S. Mezzasoma, H. Hauschildt, M. Krassenburg, and I. Shurmer, "Alphasat-Sentinel-1A optical inter-satellite links: run-up for the European data relay satellite system," in Proc. SPIE, 2016, vol. 9739, pp. 973902-973902-6.

[2] T. Jono, Y. Takayama, N. Perlot, D. Giggenbach, and others, "Report on DLR-JAXA Joint Experiment: The Kirari Optical Downlink to Oberpfaffenhofen (KIODO)," JAXA, ISSN, pp. 1349-1121, 2007.

[3] A. Biswas, B. Oaida, K. S. Andrews, J. M. Kovalik, M. Abrahamson, and M. W. Wright, "Optical payload for lasercomm science (OPALS) link validation during operations from the ISS," in Proc. SPIE, 2015, vol. 9354.

[4] C. Fuchs, D. Kolev, F. Moll, A. Shrestha, M. Brechtelsbauer, F. Rein, C. Schmidt, M. Akioka, Y. Munemasa, H. Takenaka, and others, "SOTA OPTICAL DOWNLINKS TO DLR?S OPTICAL GROUND STATIONS," 2016.

[5] D. M. Boroson, B. S. Robinson, D. V. Murphy, D. A. Burianek, F. Khatri, J. M. Kovalik, Z. Sodnik, and D. M. Cornwell, "Overview and results of the Lunar Laser Communication Demonstration," in Proc. SPIE, 2014, vol. 8971.

[6] C. Fuchs, C. Schmidt, J. Keim, F. Moll, B. Rödiger, M. Lengowski, S. Gaißer, and D. Giggenbach, "Update on DLR's OSIRIS program and first results of OSIRISv1 on Flying Laptop," in Free-Space Laser Communications XXXI, 2019, vol. 10910, p. 109100S.

[7] PC/104 Embedded Consortium, PC/104 Specification Version 2.6. 2008.

[8] L. Grillmayer and S. Arnold, "Integrating the Cubesat Space Protocol into GSOC's Multi-Mission Environment," in Small Satellite Conference, 2020.

[9] C. Stangl, A. Braun, and M. P. Geyer, "GECCOS-the new Monitoring and Control System at DLR-GSOC for Space Operations, based on SCOS-2000," in SpaceOps 2014 Conference, 2014, p. 1602. 